\title{
The mass miniature chest radiography programme in Cape Town, South Africa, 1948 - 1994: The impact of active tuberculosis case finding
}

\author{
S M Hermans, ${ }^{1,2,3} \mathrm{MD}, \mathrm{MSc}, \mathrm{PhD} ; \mathbf{J}$ R Andrews, ${ }^{4} \mathrm{MD}$, SM; L-G Bekker, ${ }^{1,5}$ FCP (SA), PhD; R Wood ${ }^{1,5,6}$ FCP (SA), DSc (Med) \\ ${ }^{1}$ Desmond Tutu HIV Centre, Institute for Infectious Diseases and Molecular Medicine, University of Cape Town, South Africa \\ ${ }^{2}$ Department of Global Health, Academic Medical Center, University of Amsterdam, Amsterdam Institute for Global Health and Development, \\ Netherlands \\ ${ }^{3}$ Department of Internal Medicine, School of Medicine, Makerere University College of Health Sciences, Kampala, Uganda \\ ${ }^{4}$ Division of Infectious Diseases and Geographic Medicine, Stanford University School of Medicine, Calif., USA \\ ${ }^{5}$ Department of Medicine, Faculty of Health Sciences, University of Cape Town, South Africa \\ ${ }^{6}$ Department of Clinical Research, Faculty of Infectious and Tropical Diseases, London School of Hygiene and Tropical Medicine, UK
}

Corresponding author: S M Hermans (sabine.hermans@hiv-research.org.za)

\begin{abstract}
Background. Tuberculosis (TB) control programmes rely mainly on passive detection of symptomatic individuals. The resurgence of TB has rekindled interest in active case finding. Cape Town (South Africa) had a mass miniature radiography (MMR) screening programme from 1948 to 1994.

Objective. To evaluate screening coverage, yield and secular trends in TB notifications during the MMR programme.

Methods. We performed an ecological analysis of the MMR programme and TB notification data from the City of Cape Town Medical Officer of Health reports for 1948 - 1994.

Results. Between 1948 and 1962, MMR screening increased to $12 \%$ of the population per annum with yields of 14 cases per 1000 X-rays performed, accounting for $>20 \%$ of total annual TB notifications. Concurrent with increasing coverage (1948 - 1965), TB case notification decreased in the most heavily TB-burdened non-European population from 844/100 000 population to 415/100 000. After 1966, coverage declined and TB notifications that initially remained stable (1967 - 1978) subsequently increased to 525/100 000. MMR yields remained low in the European population but declined rapidly in the non-European population after 1966, coincidental with forced removals from District 6. An inverse relationship between screening coverage and TB notification rates was observed in the non-European adult population. Similar secular trends occurred in infants and young children who were not part of the MMR screening programme.

Conclusion. MMR of a high-burdened population may have significantly contributed to TB control and was temporally associated with decreased transmission to infants and children. These historical findings emphasise the importance of re-exploring targeted active case finding strategies as part of population TB control.
\end{abstract}

S Afr Med J 2016;106(12):1263-1269. DOI:10.7196/SAMJ.2016.v106i12.10744

South Africa (SA) has the highest per capita rate of tuberculosis (TB) of any major country in the world, resulting in approximately $1 \%$ of the population developing TB disease annually. ${ }^{[1]} \mathrm{TB}$ is the leading cause of death in SA and is a major public health problem. ${ }^{[2]}$ The city of Cape Town has more TB notifications each year than the USA, Canada, the UK and France combined. ${ }^{[1]}$

Approaches to TB control and case finding have changed throughout the last century. ${ }^{[3]}$ Before effective chemotherapy became available in the 1950s, TB control was based on the Edinburgh Scheme pioneered by Sir Robert Phillip and implemented throughout the British Empire and colonies. Cases, which were self-referred or identified by health visitors, were assessed in a TB dispensary followed by sanatorium treatment for early cases and hospitalisation of late cases. ${ }^{[4]}$ After the development of combination chemotherapy, a strategy of indiscriminate active case finding was pursued in the 1950s in many industrialised cities by means of mass miniature radiography (MMR). In 1974, the World Health Organization (WHO) recommended discontinuing active case finding with MMR following a review of costs and low diagnostic yields reported from industrialised settings. ${ }^{[5]}$ It was also argued that MMR in settings with a high prevalence of TB imposed too high a burden on already stretched health services because it required maintenance of complex, expensive equipment and a supply of well-trained technicians. Following these WHO recommendations, enthusiasm for MMR declined and population control of TB has been based primarily on supervised administration of combination short-course therapy for passively presenting cases. ${ }^{[6]}$

The HIV pandemic and global resurgence of TB since the 1990s has led to a reassessment of the role of active case finding as a TB control measure. A 2013 WHO report ${ }^{[7]}$ assessed the contribution of systematic screening as a TB control modality, noting that the evidence for lack of effectiveness reviewed in the 1974 report was derived largely from industrialised populations. It was felt that the historical evidence derived primarily from low-prevalence settings was insufficient to predict the impact of active screening in currently high-burdened settings. Additionally, a recent review of the active case-finding literature concurred that the individual and communitylevel benefits from active screening for TB disease remain uncertain. ${ }^{\left[{ }^{[8]}\right.}$

However, mathematical modelling has shown that transmission during subclinical TB disease may be an important impediment to current $\mathrm{TB}$ control measures, a finding that supports a re-evaluation of active screening. ${ }^{[9]}$ 
Cape Town's MMR screening programme was in existence from 1948 to 1994. This time period spanned the end of the sanatorium era, the advent of chemotherapy and the subsequent focus on supervised treatment of passively presenting cases. In view of the paucity of data concerning the outcomes and impact of mass population screening from high-prevalence settings, we analysed the annual proportion of the population screened, the yield and secular trends in TB notifications during the MMR programme.

\section{Methods}

\section{The MMR screening programme}

The Cape Town TB control programme installed its first $70 \mathrm{~mm}$ MMR screening facility at the central Tuberculosis Clinic on Chapel Street in April 1948. ${ }^{[10]}$ It was a free service offered to all Capetonians. Employers, trade unions and industrial councils were actively contacted to solicit the attendance of their employees. The screening took place during working hours, appointments were organised for groups of 60 workers at a time, and transport was offered to and from their place of work. Children were not routinely screened. Those with abnormalities on MMR were recalled for evaluation including sputum smear microscopy, tuberculin skin testing (TST) and a large-film chest radiograph. If the person was diagnosed with $\mathrm{TB}$, they would be referred for treatment to their nearest $\mathrm{TB}$ clinic. Streptomycin monotherapy became available in 1948 and combination chemotherapy in $1953 .^{[10]}$

Publicity for the MMR service was offered by the local press and the South African Broadcasting Corporation. In addition, a locally made colour film was screened in social agencies and clubs, and to factory groups and welfare organisations. Attendance was voluntary, but was facilitated by the refusal to screen factory groups unless the volunteer rate was $>80 \%$. Initially the screening was offered annually. From 1985 onwards, routine annual screening for all was no longer encouraged and screening of workers before employment and of high-risk groups was prioritised.

A second MMR facility was installed in Langa township in 1967 for the pre-employment screening of newly arriving workers from the rural areas (Fig. 1). The facility was burnt down in the civil unrest of 1976 , after which it was never fully restored. It was taken out of use in 1986.

\section{Data sources}

The estimated mid-year populations of the City of Cape Town from 1948 to 1994 were extracted from the Medical Officer of Health

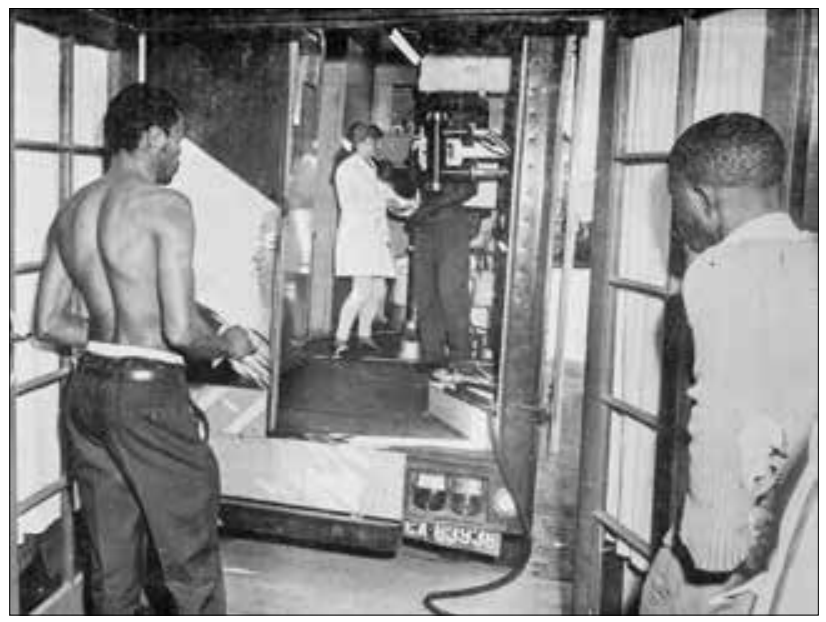

Fig. 1. Radiographic screening of migrant workers in Langa, Cape Town (source $\mathrm{MoH}^{[10]}$ ).
$(\mathrm{MoH})$ annual reports. ${ }^{[10]}$ The number of miniature chest radiographs performed, the radiology location, the resultant large-film chest radiographs and $\mathrm{TB}$ case notifications were also obtained from the $\mathrm{MoH}$ annual reports. ${ }^{[10]}$ Results of sputum smear microscopy and TST were not available. The total city TB notifications were recorded, but their method of diagnosis was not consistently reported. Data were available stratified by population group (European and nonEuropean) until 1989, except for data on TB diagnoses, which were only available until 1979. Data stratified by age were available throughout.

\section{Statistical methods}

Diagnostic yields per 1000 radiographs were calculated using the numbers of TB notifications from the MMR services and the radiographs performed at that service annually. The annual proportion of the population screened was calculated by dividing the number of people screened by MMR by the estimated mid-year population. The contribution to TB notification was calculated by dividing the number of TB diagnoses by MMR (excluding those already known to the TB programme) by the number of TB notifications in that year. Annual TB notification rates per 100000 population were calculated using TB notifications and mid-year population estimates from 1948 to 1994. Notification rates and the proportions screened were also calculated stratified by population group and in 0 - 4-yearolds, as TB in this age group reflects recent transmission. Only data from the Chapel Street screening unit were used for calculation of the screening coverage, the contribution to TB notification or TB notification rates. The Langa unit screened migrant workers who were not included in the Cape Town population estimates, and any TB diagnoses were considered imported infections and therefore not included in the Cape Town notifications. All analyses were done using Microsoft Excel, 2010 (USA).

\section{Ethical considerations}

This analysis was performed using publicly available data, which are considered exempt from research ethics review according to the Standard Operating Procedures of the University of Cape Town Human Research Ethics Committee.

\section{Results \\ Numbers of radiographs performed}

Between 1948 and 1994, the Cape Town TB control programme performed approximately 2.6 million mass miniature radiographs, during which time the population grew from 400000 to 1300000 . The majority of radiographic screens $(87 \%)$ were performed at the Chapel Street clinic. Men were screened more often than women, ranging between $53 \%$ and $72 \%$ of those screened.

Between 1967 and 1985, the Langa unit screened 324000 black workers, who were largely from the rural Eastern Cape. The annual numbers of radiographs performed in the two services are shown in Fig. 2.

\section{Diagnostic yield}

The proportion of people screened who were recalled for a subsequent large film in Chapel Street declined from 7\% in 1951 to $1 \%$ in 1978, after which it increased to $5 \%$ in 1994 . Over the entire duration of the MMR programme a total of 17890 TB cases were identified: 12725 at the Chapel Street clinic and 5181 at the Langa clinic. From 1948 to 1966, the TB diagnostic yield at Chapel Street ranged between 6 to 14 cases per 1000 radiographs performed. From 1966 to 1980, the yield steadily declined from 5 to 1 per 1000 , after which it increased again to 6 per 1000 . The yield was consistently higher among nonEuropeans compared with Europeans (Fig. 3). As a result, screening 


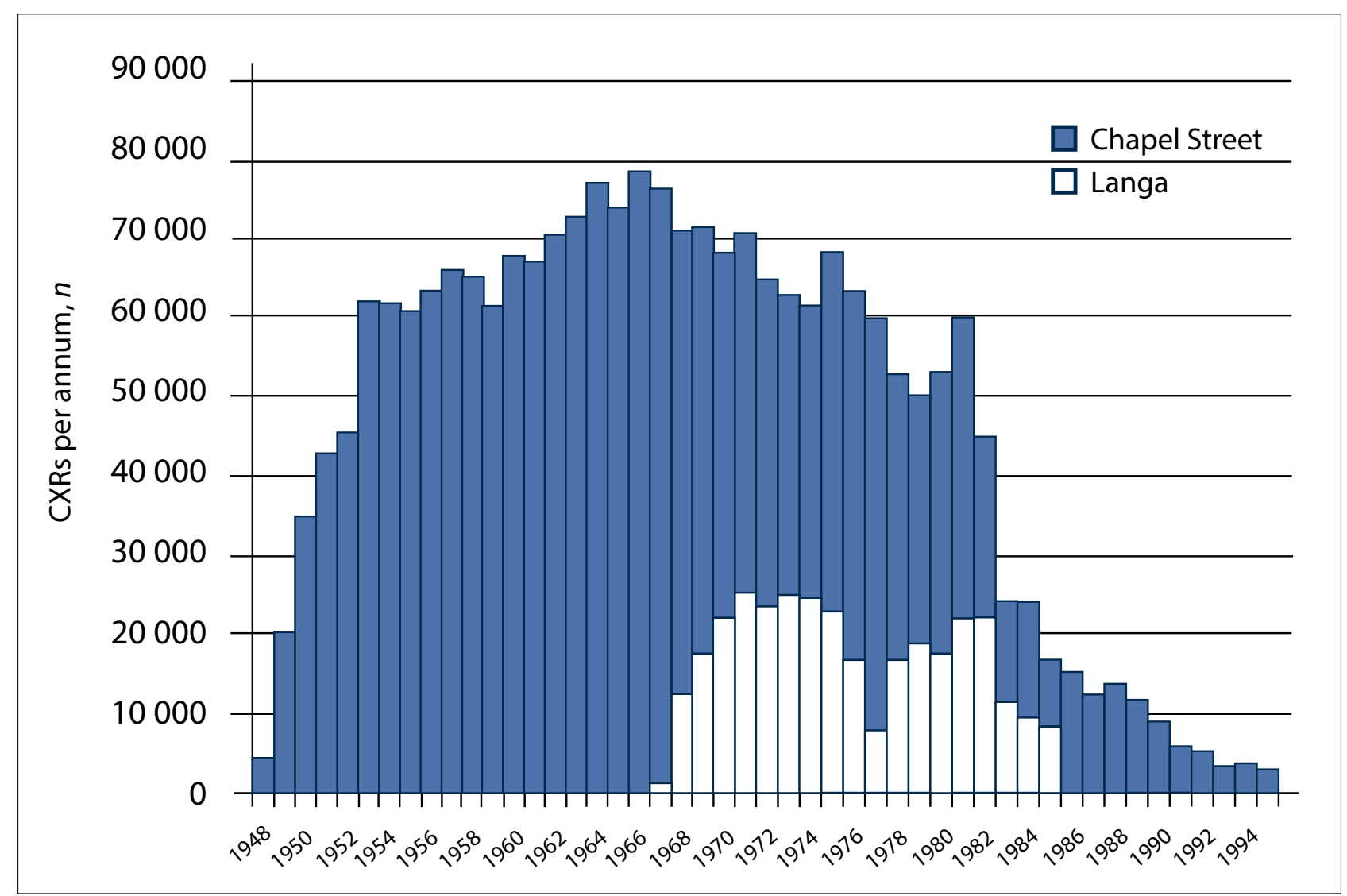

Fig. 2. Annual numbers of TB screening chest radiographs performed in the Chapel Street and Langa services (the columns overlap). (CXRs = chest radiographs.)

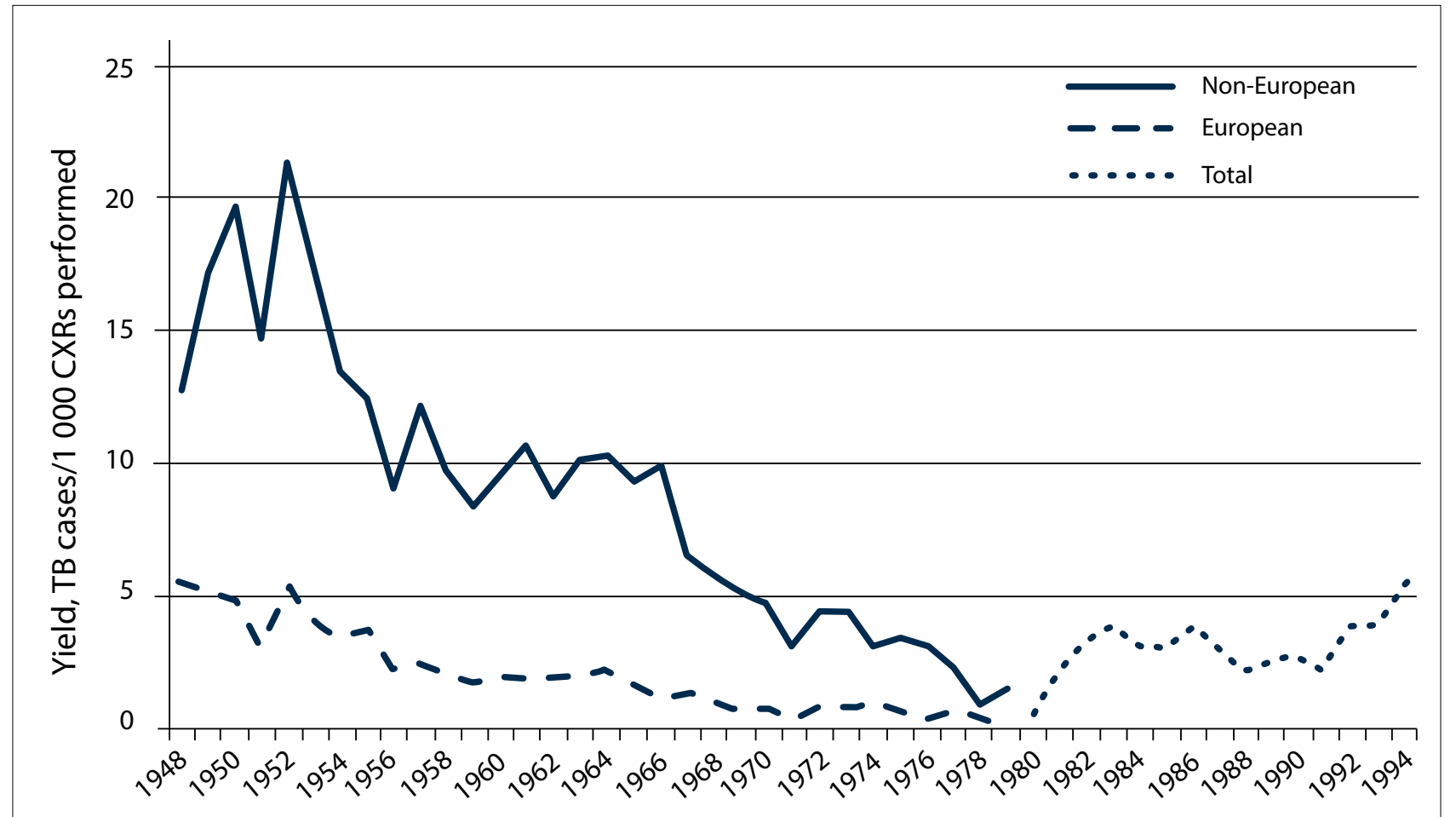

Fig. 3. Diagnostic yield of chest radiographs from the Chapel Street clinic over time. Results were stratified by population group until 1979, after which stratified data were no longer available. (CXRs = chest radiographs.)

of Europeans was not deemed cost-effective and screening policy was changed, leading to a reducing proportion of screens of Europeans compared with non-Europeans from $50 \%$ to $11 \%$.
During the two decades of the Langa service, the annual diagnostic yield gradually decreased from 40 per 1000 radiographs to 6 per 1000. 


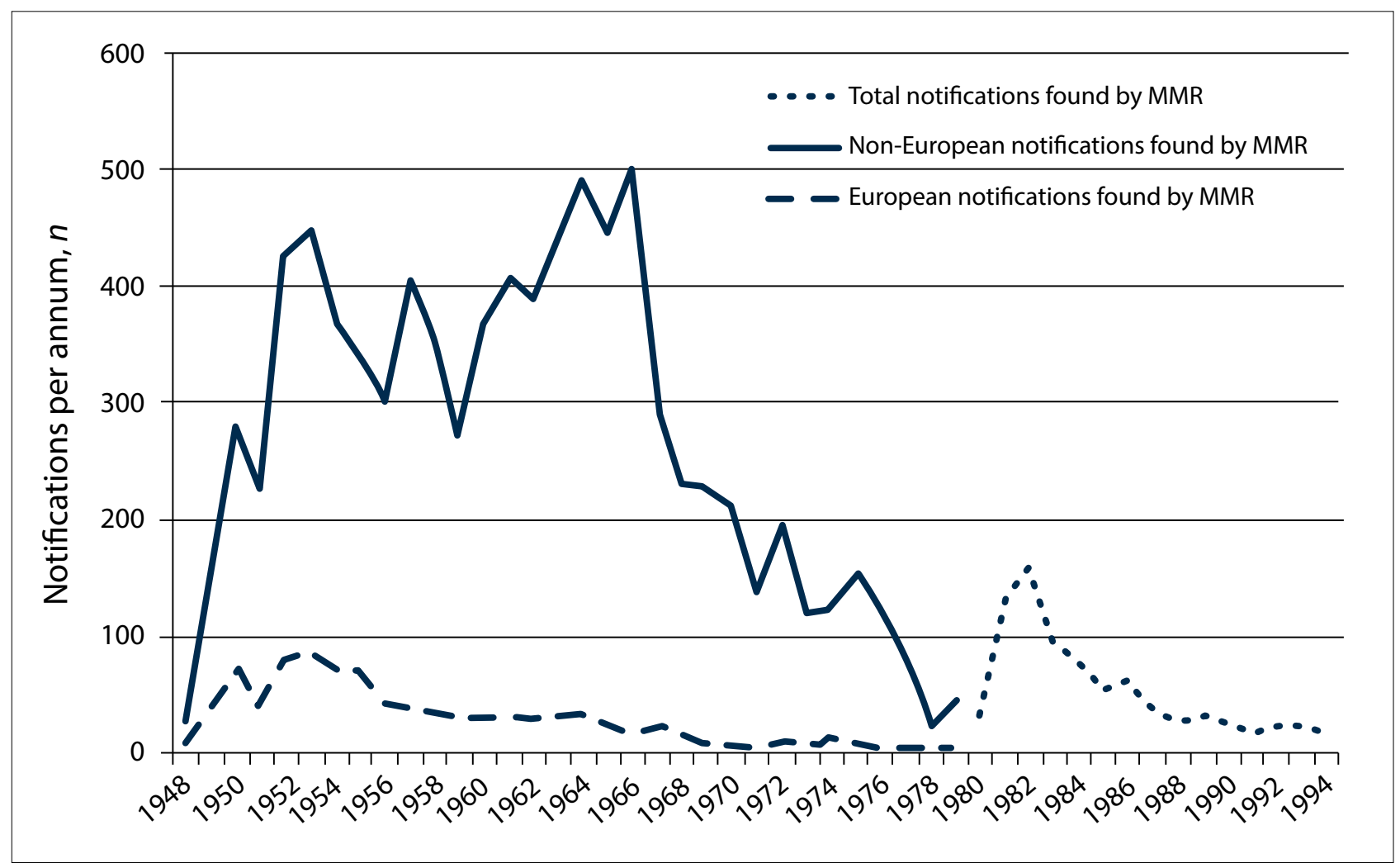

Fig. 4. Number of city TB notifications identified by the MMR service over time (Chapel Street clinic). Numbers were stratified by population group until 1979, after which stratified data were no longer available.

\section{The contribution to TB notification}

The majority of the annual reports noted that many of the diagnosed cases were asymptomatic and unsuspected, but did not enumerate this. A substantial proportion of the Chapel Street cases found were already known to the $\mathrm{TB}$ programme, ranging from $0 \%$ to $36 \%$ without any clear trend over time. The number of TB cases identified via MMR was consistently higher among non-Europeans than Europeans, and dropped substantially after 1966 (Fig. 4).

The percentage of total annual TB cases notified in Cape Town found by MMR screening increased from 2\% in 1948 to a peak of $24 \%$ in 1962, after which it declined to $0.4 \%$ in 1994 . This percentage was more or less equal in the two population groups.

\section{Screening coverage and impact on TB notification rate}

The proportion of the population screened increased rapidly to a maximum of 14\% between 1953 and 1957, after which it decreased to $0.3 \%$ in 1994 . This decrease started earlier in the European than in the non-European population (1954 v. 1966) and was more rapid (Fig. 5, A and B).

The TB notification rate declined from 521/100 000 in 1948 to 275/100 000 in 1960. From 1960 to 1978 the rate fluctuated around this level, after which it increased again. This pattern was only seen in the non-European population; TB notification rates in the European population were much lower and decreased throughout the study period (Fig. 5, A and B). In the non-European population, rates among children aged $<5$ years decreased by $72 \%$ to a nadir in 1970 , during which period the overall rates decreased by $57 \%$. Childhood rates started increasing around 5 years earlier than the overall rates and rose to a lesser extent than in adults (Fig. 6).

There appeared to be an inverse relationship between the proportion of the population screened and the $\mathrm{TB}$ notification rate in the non-European population: screening coverage was over $14 \%$ in the period of decreasing rates, decreasing from $14 \%$ to $8 \%$ when rates were at their lowest, and dropping below $8 \%$ when rates started increasing again.

\section{Discussion}

To our knowledge, this is the first report of the outcomes of a mass radiography $\mathrm{TB}$ screening programme in a TB-endemic setting in Africa. Over almost half a century, annual screening numbers and diagnostic yields varied considerably. In its heyday in the 1950s, the programme screened almost a quarter of the estimated working population annually and found up to a fifth of the annual TB cases notified in the city.

After the mid-1960s the diagnostic yield at the Chapel Street clinic rapidly decreased, coincidental with a large population movement from the central city. Forced removals of the population of the nearby poor residential area of District Six started on 11 February 1966, resulting in approximately 60000 relocations by 1982 . From the end of the 1970s onwards, even though the city TB notification rates were increasing, the diagnostic yields of the services were consistently low, probably as a result of a failure to target the high-burdened segments of the population. The apartheid regime may have decreased the effectiveness of the MMR programme and therefore negatively affected TB control in the city.

Up to $20 \%$ of notifications in both the non-European and European population were found by MMR screening, indicating that a relatively asymptomatic but chest X-ray-positive proportion of cases occurred in both high- and low-burden populations. However, the incidence of TB did not rebound rapidly in the European population after its discontinuation. The lack of impact of screening in the European population of Cape Town is probably similar to the 


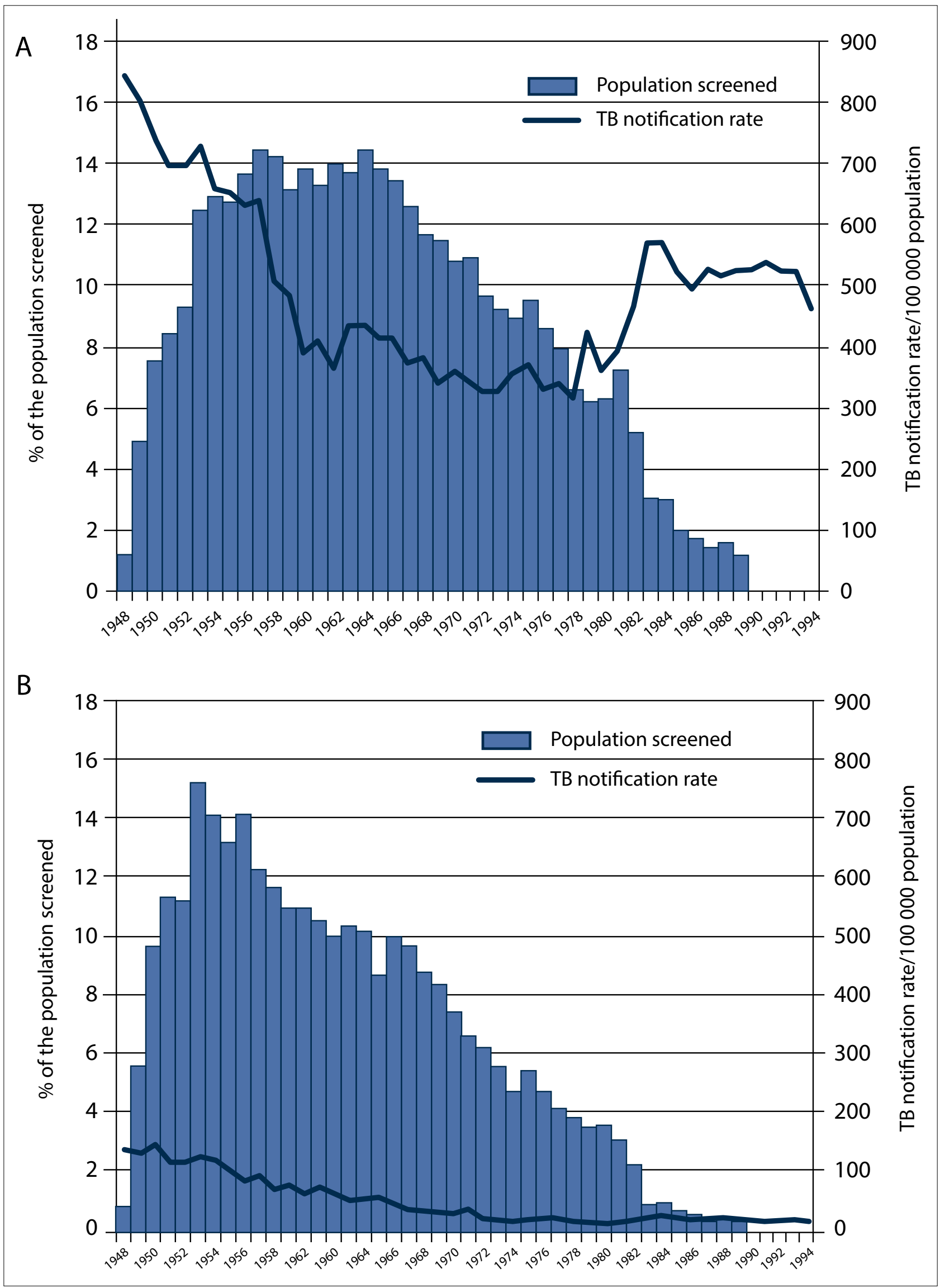

Fig. 5. Annual TB notification rates (/100 000 population) in comparison with the percentage of the population screened. (A) Non-European population; (B) European population. 


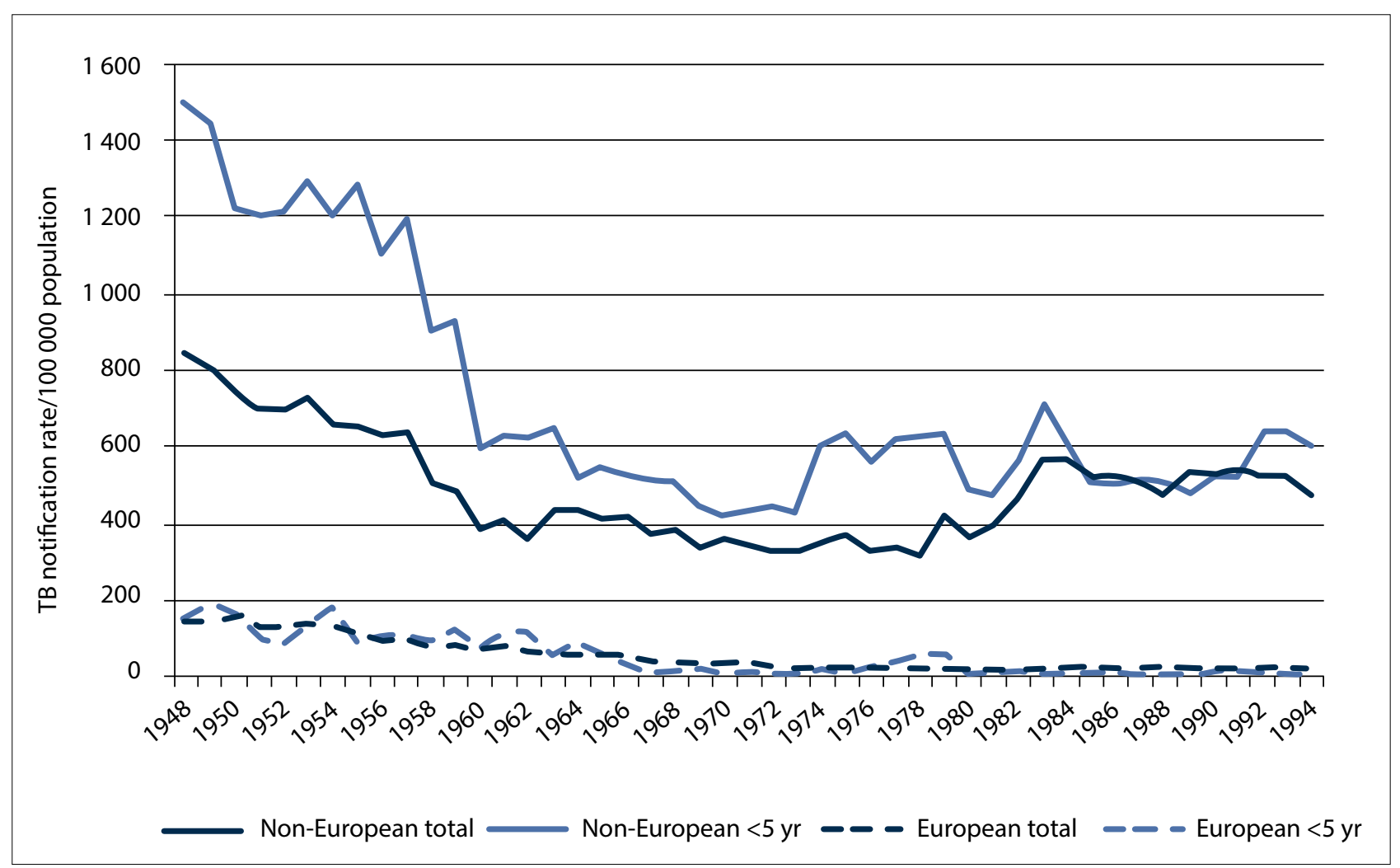

Fig. 6. Annual TB notification rates (/100 000 population) in the overall population and among children aged $<5$ years, stratified by population group.

experience of mass screening in industrialised countries, which led to the discontinuation of this type of screening.

However, our data suggest an effect of screening on the highburdened, non-European TB notification rates: these started to increase when MMR coverage fell $<8 \%$. As screening coverage had been decreasing gradually, it could be hypothesised that a screening coverage threshold exists above which TB transmission is affected. A time lag between screening and the impact on the TB notification rate is likely, but could not be determined from our data. However, disease rates among young children can offer a proxy for recent transmission. We found larger decreases in TB rates among non-European infants and young children, who were not part of the MMR screening programme. Our data were compatible with decreasing transmission rates during the period of maximum screening. This inverse relationship could be a biologically plausible impact of active TB case finding. However, causality is not proven by temporal association, as other unrecognised confounders may have been present. Neonatal BCG vaccination could have been such a confounder; however, it was introduced in 1959, when rates among young children and infants had already begun to decline. ${ }^{[8]}$

Prospective assessment of the effectiveness of screening is difficult, influenced by an underlying changing epidemic, and a successful programme can become 'victim of its own success'. Formal statistical testing of an association between the proportion of the population screened and the TB notification rate was precluded by variable co-dependencies and the non-stationarity of their relationship. Additionally, our analysis would have underestimated the population impact of the screening programme by excluding extra-municipal cases identified in the Chapel Street clinic. As these people worked in Cape Town, they would certainly have contributed to local TB transmission.
There are many confounding factors that complicate the interpretation of the impact of screening in our ecological analysis. Combination TB treatment became routinely available in $1953,,^{[10,11]}$ which could have also led to a shortening of the duration of infectiousness and therefore a reduction in population $\mathrm{TB}$ incidence. Later, a reduction in socioeconomic circumstances associated with the living conditions in the newly populated townships could have contributed to the increase in the TB notification rate. Despite the rapid growth of the city during this time, the TB services seem to have expanded sufficiently to provide adequate care. Other practices in the TB control programme may have changed during this time, including contact tracing and follow-up of those on treatment, although this was not apparent in the detailed $\mathrm{MoH}$ reports. The increasing rates in the early 1980s are unlikely to have been due to the HIV epidemic, which began much later in Cape Town than in developed countries and matured in the mid- to late $1990 \mathrm{~s}^{[12]}$

The Langa screening unit had a consistently higher yield than the Chapel Street clinic. As the population screened there were workers newly arrived in the township, the results may better reflect the TB epidemic conditions of the rural areas from where the workers were recruited. The consistently high yields reinforce the need of screening services to target the most affected segments of society in order to achieve the highest diagnostic yields.

In the current pandemic situation, SA urgently needs new strategies to control the transmission of TB. The evidence base for active case finding as such a strategy in similar high-burden settings is limited, ${ }^{[8]}$ although there have been some promising results regarding the impact on earlier case detection and population TB incidence. ${ }^{[13,14]}$ This study adds to these limited data on effectiveness, and even more sparse data on mass radiography. Our data highlight that active case 
finding among a large proportion of the population was feasible, and had potential to be effective. Novel strategies for active case finding need to be considered, such as digital X-rays and newer molecular diagnostic techniques. The cost-effectiveness of active case finding depends on the method and frequency of screening, and the baseline prevalence and incidence of disease. ${ }^{[15]}$

While acknowledging the limitations in evaluating causation from observational data, the period when population screening exceeded $10 \%$ was temporally associated with a decline in TB notification rates. In the most burdened population, high MMR screening was temporally associated with a decline in the annual TB notification rate. The decline in childhood $\mathrm{TB}$, a proxy for recent transmission, was even more marked. These historical findings emphasise the importance of re-exploring targeted active case-finding strategies for TB control in high-burdened settings.

Acknowledgements. We thank Allan Clark for his help with exploring the use of time-series analysis methodology with our data, and Edwin Hermans for the editing of Fig. 1. We are grateful to the team at the University of Cape Town Health Sciences Library for their co-operation. Funding. This work was supported by the European Union (Marie Curie International Outgoing Fellowship for Career Development PIOFGA-2012-332311 to SH), the South African Medical Research Council (MRC-RFAUFSP-01-2013/CCAMP to RW), the National Institutes of Health (K01AI104411 to JRA; R01AI058736 and R01AI093269 to RW) and the Bill \& Melinda Gates Foundation (OPP1116641 to RW). The content is solely the responsibility of the authors and does not necessarily represent the official views of the funders.
Author contributions. SH and RW conceived and designed the study and collected the data. SH and JA analysed the data. All authors interpreted the data and critically reviewed the manuscript. SH wrote the first draft.

1. World Health Organization. Report on Global Tuberculosis Control. Geneva: WHO, 2015.

2. Statistics South Africa. Mortality and Causes of Death in South Africa, 2013: Findings from Death Notification. Report No. 2-12-14. Pretoria: SSA, 2014

3. Golub JE, Mohan CI, Comstock GW, Chaisson RE. Active case finding of tuberculosis: Historical perspective and future prospects. Int J Tuberc Lung Dis 2005;9(11):1183-203.

Dubovsky H. Robert W. Philip (1857-1939), pioneer of the holistic approach to tuberculosis and its voluntary movement. S Afr Med J 1973;47(23):1007-1010.

5. World Health Organization. WHO Expert Committee on Tuberculosis, Ninth Report. Technical Report Series No. 552. Geneva: WHO, 1974.

6. Raviglione MC, Pio A. Evolution of WHO policies for tuberculosis control, 1948-2001. Lancet 2002;359(9308):775-780. http://dx.doi.org/10.1016/S0140-6736(02)07880-7

7. World Health Organization. Systematic Screening for Active Tuberculosis. Principles and Werld Health Organization. Systematic

8. Kranzer K, Afnan-Holmes $\mathrm{H}$, Tomlin $\mathrm{K}$, et al. The benefits to communities and individuals of
8. Kranzer K, Afnan-Holmes H, Tomlin K, et al. The benefits to communities and individuals of
screening for active tuberculosis disease: A systematic review. Int J Tuberc Lung Dis 2013;17(4):432screening for active tuberculosis disease: A

9. Dowdy DW, Basu S, Andrews JR. Is passive diagnosis enough? The impact of subclinical disease on diagnostic strategies for tuberculosis. Am J Respir Crit Care Med 2013;187(5):543-551. http://dx.doi. org/10.1164/rccm.201207-12170

10. Medical Officer of Health, City of Cape Town. Annual Reports 1904-1994.

11. Hermans S, Horsburgh CR Jr, Wood R. A century of tuberculosis epidemiology in the northern and southern hemisphere: The differential impact of control interventions. PloS One 2015;10(8):e0135179. http://dx.doi.org/10.1371/journal.pone.0135179

12. Actuarial Society of South Africa. AIDS and Demographic Model 2008. Cape Town: ASSA, 2011. http://www. actuarialsociety.org.za/Societyactivities/CommitteeActivities/DemographyEpidemiologyCommittee/ Models.aspx (accessed 29 September 2016).

13. Corbett EL, Bandason T, Duong T, et al. Comparison of two active case-finding strategies for communityCorbett EL, Bandason T, Duong T, et al. Comparison of two active case-finding strategies for community-
based diagnosis of symptomatic smear-positive tuberculosis and control of infectious tuberculosis in based diagnosis of symptomatic smear-positive tuberculosis and control of infectious tuberculosis in
Harare, Zimbabwe (DETECTB): A cluster-randomised trial. Lancet 2010;376(9748):1244-1253. http:// Harare, Zimbabwe (DETECTB): A cluster-r
dx.doi.org/10.1016/S0140-6736(10)61425-0

14. Churchyard GJ, Fielding K, Roux S, et al. Twelve-monthly versus six-monthly radiological screening for active case-finding of tuberculosis: A randomised controlled trial. Thorax 2011;66(2):134-139. http://dx.doi.org/10.1056/NEJMoa1214289

15. Dodd PJ, White RG, Corbett EL. Periodic active case finding for TB: When to look? PloS One 2011;6(12):e29130. http://dx.doi.org/10.1371/journal.pone.0029130

Accepted 16 March 2016 